\title{
Quantum Recurrences in Driven Power-law Potentials
}

\author{
Shahid Iqbal ${ }^{1}$, Qurat-ul-Ann ${ }^{1}$ and Farhan Saif ${ }^{1,2 *}$ \\ 1 Department of Electronics, Quaid-i-Azam University, Islamabad 45320, \\ Pakistan. \\ 2 Department of Physics and Astronomical Sciences, The University of Ari- \\ zona, Tucson 85721, Arizona, USA. \\ * saif@physics.arizona.edu,saif@fulbrightweb.org
}

\begin{abstract}
The recurrence phenomena of an initially well localized wave packet are studied in periodically driven power-law potentials. For our general study we divide the potentials in two kinds, namely tightly binding and loosely binding potentials. In the presence of an external periodically modulating force, these potentials may exhibit classical and quantum chaos. The dynamics of a quantum wave packet in the modulated potentials displays recurrences at various time scales. We develop general analytical relations for these times and discuss their parametric dependence.
\end{abstract}

PACS numbers: 05.45.-a, 03.65.-w, 45.80.+r, 47.52.+j 


\section{Introduction}

In one degree of freedom systems, wave packet dynamics manifests quantum recurrences $[1,2,3,4,5,6,7,8,9]$. An initially well-localized wave packet in a bounded system follows classical evolution in its short time dynamics and displays reconstruction after a classical period. However, after many classical periods phase difference between constituent wavelets develops which leads to destructive interference and results a collapse when the phase difference is maximum. Later, constructive interference dominates and supports quantum revivals and fractional revivals. In this paper we study a general class of one degree of freedom bounded systems defined by power-law potentials and show that the quantum recurrence phenomena occurs in the presence of external periodic forces.

The quantum recurrences have been studied in a variety of quantum mechanical systems, such as, in the Jaynes-Cummings model of the quantum electrodynamics $[10,11]$, in the micromaser cavity [12], in the Rydberg wave packets [13], and in the multi-atomic molecules [14]. Quantum recurrences in periodically driven quantum chaotic systems $[15,16,17,18]$ and in general higher dimensional systems are proved to be generic $[19,20]$. The phenomena is vital to extend the horizons of nanotechnology and to develop newer experimental techniques to study surface structures with atomic size resolution $[21]$.

In this paper, we introduce a general class of potentials as power-law potentials $[22,23,24,25]$ and calculate the quantum recurrence times in 
the presence of external periodic force. We develop analytical relations for recurrence times, namely the classical period and the quantum revival time, and explain their parametric dependence. Our analytical treatment leads us to explain the interdependence of these times in the loosely binding and the tightly binding power law potentials as well.

The paper is organized as follows. In Sec. II., we classify the general power law potentials and write the general Hamiltonian and corresponding quantized energy for the system. In Sec. III., we calculate the general relations for the classical period and the quantum revival time in the power-law potentials. In Sec. IV., we explain the dependence of time scales on nonlinearity-measure parameter. Later, we extend the discussion to tightly binding potentials and loosely binding potentials in Secs. V. and VI., respectively.

\section{Classification of potentials}

On the basis of their energy spectrum, we classify potentials as of two kinds: i) Tightly binding potentials, for which level spacing between the adjacent energy levels increases as the quantum number increases. ii) Loosely binding potentials, for which the level spacing between adjacent levels reduces as the principle quantum number increases. We may express the two kinds of the potentials mathematically by a general power law expression, viz.,

$$
\tilde{V}_{(k)}(X)=\tilde{V}_{0}\left|\frac{X}{a}\right|^{k},
$$


where, $\tilde{V}_{0}$ and $a$ are constants with the dimensions of energy and length, respectively. Moreover, $k$ defines the power-law exponent. For the exponent $k>2$, we find tightly binding potentials and $k<2$ expresses loosely binding potentials.

In the presence of an external time periodic force, the dynamics in powerlaw potentials is governed by the general Hamiltonian given as

$$
\tilde{H}(X, P, \tau)=\tilde{H}_{0}(X, P)+\lambda \tilde{V}(X) \sin \Omega \tau
$$

where, $\tilde{H}_{0}(X, P)=\frac{P^{2}}{2 m}+\tilde{V}_{0}\left|\frac{X}{a}\right|^{k}$, describes the unmodulated system. In the second term $\lambda$ expresses the dimensionless external modulation strength.

With the help of WKB approximation we express the energy quantization in the unmodulated system $[22,23,24,25]$ as

$$
\tilde{E}_{n}^{(k)}=\left[\left(n+\frac{\gamma}{4}\right) \frac{\hbar \pi}{2 a \sqrt{2 m}} \tilde{V}_{0}^{1 / k} \frac{\Gamma(1 / k+3 / 2)}{\Gamma(1 / k+1) \Gamma(3 / 2)}\right]^{2 k /(k+2)},
$$

here, $n$ is the corresponding principle quantum number. Moreover, $\gamma$ defines the Maslov index, which takes integer values depending on the boundary conditions at the turning points. For example, in the case of harmonic oscillator we have $\gamma=2$ and in case of one dimensional box we find $\gamma=4$.

Equation (3) leads us to conclude that the energy difference between adjacent levels, $\Delta E_{n}=E_{n}-E_{n-1} \propto\left(n+\frac{\gamma}{4}\right)^{\frac{k-2}{k+2}}$, increases for $k>2$ as the quantum number $n$ increases. Therefore, Eq. (1) defines tightly binding potentials for $k>2$. In contrast, for $k<2$ the energy difference between adjacent levels, $\Delta E_{n}=E_{n}-E_{n-1} \propto\left(n+\frac{\gamma}{4}\right)^{\frac{k-2}{k+2}}$, decreases with the increase 
of the principle quantum number $n$. For the reason, Eq. (1) defines loosely binding potentials for $k<2$.

In order to simplify our calculations we introduce the dimensionless position coordinate, $x=\frac{X}{a}$, and dimensionless momentum coordinate, $p=\frac{P}{\sqrt{m \hbar \Omega}}$. The commutation relation between $x$ and $p$ leads us to find out an effective Planck's constant, $k$, such that,

$$
[x, p]=\frac{1}{a \sqrt{m \Omega \hbar}}[X, P]=i \hbar,
$$

where, $k=\frac{1}{a} \sqrt{\frac{\hbar}{m \Omega}}$. The scaled position and momentum coordinates help us to scale the general Hamiltonian given in Eq. (2), and we find

$$
H(x, p, t)=\frac{p^{2}}{2}+V_{0}|x|^{k}+\lambda V(x) \sin t \equiv H_{0}(x, p)+\lambda V(x) \sin t,
$$

where, $H(x, p, t)=\tilde{H}(X, P, \tau) / \hbar \Omega$, and $t=\Omega \tau$. Moreover, the amplitude $V_{0}=\tilde{V}_{0} / \hbar \Omega$ and the spatial component of external modulation $V(x)=$ $\tilde{V}(X) / \hbar \Omega$. In the dimensionless form the quantized energy, given in Eq. (3), becomes

$$
E_{n}^{(k)}=\left[\left(n+\frac{\gamma}{4}\right) \frac{k \pi}{2 \sqrt{2}} V_{0}^{1 / k} \frac{\Gamma(1 / k+3 / 2)}{\Gamma(1 / k+1) \Gamma(3 / 2)}\right]^{2 k /(k+2)},
$$

where, $E_{n}^{(k)}=\tilde{E}_{n}^{(k)} / \hbar \Omega$, is the scaled energy.

\section{Quantum Recurrences in Power law po- tentials}

In the periodically driven power-law potentials energy is no longer a constant of the motion. For the reason we solve the time dependent Schrödinger 
equation by using secular perturbation approximation as suggested by Max Born [26]. This leads us to find the solution by averaging over rapidly changing variable and we, therefore, find out a partial solution of the periodically driven system. The procedure provides us the quasi eigen energy states and the quasi eigen energy values for the nonlinear resonances of the system.

In order to study the $N$ th quantum nonlinear resonances of the system $[27,28]$, we write the solution of the Schrödinger equation, corresponding to the Hamiltonian in Eq. (5), in the form of an anstaz, viz.,

$$
|\psi(t)\rangle=\sum_{n} C_{n}(t)|n\rangle \exp \left\{-i\left[E_{\bar{n}}^{(k)}+(n-\bar{n}) \frac{k}{N}\right] \frac{t}{k}\right\} .
$$

Here, $E_{\bar{n}}^{(k)}$ is the mean energy of the wave packet in the Nth resonance.

On substituting Eq. (7) in the time dependent Schrödinger equation, we find that the probability amplitude for any nth state, $C_{n}$, changes following the equation,

$$
i \hbar \dot{C}_{n}=\left[E_{n}^{(k)}-E_{\bar{n}}^{(k)}-(n-\bar{n}) \frac{\hbar}{N}\right] C_{n}(t)+\frac{\lambda V}{2 i}\left(C_{n+N}-C_{n-N}\right) .
$$

In order to obtain Eq. (8), we average over the fast oscillating terms and keep only the resonant ones. For larger values of $n$, we consider the corresponding off-diagonal matrix elements approximately the same, so that, $V_{n, n+N} \approx$ $V_{n, n-N}=V$.

We take the initial excitation, narrowly peaked around the mean value, $\bar{n}$. For the reason, we take slow variations in the energy, $E_{n}^{(k)}$, around the $\bar{n}$ in a 
nonlinear resonance, and expand it up to second order in Taylor's expansion. Thus, the equation of motion for the probability amplitude, $C_{n}$, is

$$
i \hbar \dot{C}_{n}=k(n-\bar{n})\left(\omega-\frac{1}{N}\right) C_{n}(t)+\frac{1}{2} \hbar(n-\bar{n})^{2} \zeta C_{n}(t)+\frac{\lambda V}{2 i}\left(C_{n+N}-C_{n-N}\right) .
$$

Here, the frequency of the system $\omega$ is defined by

$$
\omega=\left.\frac{\partial E_{n}^{(k)}}{k \partial n}\right|_{n=\bar{n}}=\frac{1}{k} \frac{2 k}{k+2}\left(\bar{n}+\frac{\gamma}{4}\right)^{-1} E_{\bar{n}}^{(k)},
$$

and the nonlinearity, $\zeta$, in the time independent energy spectrum becomes

$$
\zeta=\left.\frac{\partial^{2} E_{n}^{(k)}}{k^{2} \partial n^{2}}\right|_{n=\bar{n}}=\frac{1}{k^{2}} \frac{2 k(k-2)}{(k+2)^{2}}\left(\bar{n}+\frac{\gamma}{4}\right)^{-2} E_{\bar{n}}^{(k)} .
$$

We introduce the Fourier representation for $C_{n}$ as,

$$
C_{n}=\frac{1}{2 N \pi} \int_{0}^{2 N \pi} g(\theta, t) e^{-i(n-\bar{n}) \theta / N} d \theta
$$

which helps us to express Eq. (9) as the Schrödinger equation for $g(\theta, t)$, such that $i \hbar \dot{g}(\theta, t)=H(\theta) g(\theta, t)[27,28]$. The Hamiltonian $H(\theta)$ is, therefore, given as,

$$
H(\theta)=-\frac{N^{2} k^{2} \zeta}{2} \frac{\partial^{2}}{\partial \theta^{2}}-i N k\left(\omega-\frac{1}{N}\right) \frac{\partial}{\partial \theta}-\lambda V \sin \theta .
$$

In order to obtain this equation, we consider the function $g(\theta, t)$ as $2 N \pi$ periodic, in $\theta$ coordinate.

Due to the time-independent behavior of the Hamiltonian, we can write the time evolution of $g(\theta, t)$, as $g(\theta, t)=\tilde{g}(\theta) \exp \{-i \varepsilon t / \hbar\}$. As a consequence, 
the time dependent Schrödinger equation for $g(\theta, t)$, reduces to the standard Mathieu equation,

$$
\left[\frac{\partial^{2}}{\partial z^{2}}+a_{\nu}-2 q \cos 2 z\right] \chi(z)=0 .
$$

Here, we express $\tilde{g}=\chi(z) \exp \left(-2 i\left(\omega-\frac{1}{N}\right) z / N k \zeta\right)$, where $\theta=2 z+\pi / 2$. In Eq. (14), the Mathieu characteristic parameter $a_{\nu}$, and $q$ are

$$
a_{\nu}=\frac{8}{N^{2} k^{2} \zeta}\left[\frac{\left(\omega-\frac{1}{N}\right)^{2}}{2 \zeta}+\varepsilon\right],
$$

and

$$
q=\frac{4 \lambda V}{N^{2} k^{2} \zeta}
$$

respectively. Hence, the quasi eigen energy of the system is obtained from Eq. (15) as,

$$
\varepsilon_{\nu}=\frac{N^{2} \hbar^{2} \zeta}{8} a_{\nu}-\frac{\left(\omega-\frac{1}{N}\right)^{2}}{2 \zeta},
$$

where, the index $\nu$ is $\nu=2(n-\bar{n}) / N^{2}+2(\omega-1 / N) / N k \zeta$.

The time scales, $T^{(j)}$, at which recurrences of an initially well localized wave packet occur in the driven system, depend upon the quasi eigen energy such that, $T^{(j)}=\frac{2 \pi}{\omega^{(j)}}$, where $\omega^{(j)}=(j ! k)^{-1} \partial^{j} \varepsilon_{n} / \partial n^{j}[19,20]$. Therefore, the classical period, $T^{(1)}=T_{\lambda}^{(c l)}$, and the quantum revival time, $T^{(2)}=T_{\lambda}^{(Q)}$, for the driven power-law potentials take the shape

$$
T_{\lambda}^{(c l)}=\left\{1-M_{0}^{(c l)}\right\} T_{0}^{(c l)} \Delta
$$

and

$$
T_{\lambda}^{(Q)}=\left\{1-M_{0}^{(Q)}\right\} T_{0}^{(Q)}
$$


Here, $T_{0}^{(c l)}=2 \pi / \omega$ and $T_{0}^{(Q)}=2 \pi /\left(\frac{1}{2 !} k \zeta\right)$ are the classical period and quantum revival time in the absence of external modulation. Moreover, $\Delta=\left(1-\omega_{N} / \omega\right)^{-1}$ where $\omega_{N}=1 / N$.

The time modification factors $M_{0}^{(c l)}$ and $M_{0}^{(Q)}$ are given as,

$$
M_{0}^{(c l)}=-\left\{\frac{1}{2}\left(\frac{\lambda V \zeta \Delta^{2}}{\omega^{2}}\right)^{2} \frac{1}{\left(1-\mu^{2}\right)^{2}}\right\},
$$

and

$$
M_{0}^{(Q)}=\left\{\frac{1}{2}\left(\frac{\lambda V \zeta \Delta^{2}}{\omega^{2}}\right)^{2} \frac{3+\mu^{2}}{\left(1-\mu^{2}\right)^{3}}\right\},
$$

where $\mu=N k \zeta \Delta / 2 \omega$. Here, $\omega=\left.\frac{\Delta E_{n}^{(k)}}{\hbar \Delta n}\right|_{n=\bar{n}}$ gives us the information about the energy difference between two adjacent levels and, $\zeta$ determines the nonlinearity associated with the energy spectrum corresponding to the unmodulated system.

\section{Power Law Exponent and Quantum Re- currence Times}

In section III., we calculated the classical period and the quantum revival time for the power-law potentials in the presence of external fields. As discussed earlier in section II. we may consider two kinds of power-law potentials, namely, tightly binding potentials and loosely binding potentials. The two kinds of potentials are defined in terms of power law exponent. The typical value of the exponent reflects itself in the frequency $\omega$, and in the nonlinearity, $\zeta$, present in the time independent systems, and expressed in Eqs. (10) and (11), respectively. 
We note that for the special case of the quadratic potential the power-law exponent $k$ takes the value as, $k=2$. Equation (3) provides a non-trivial result and we note that in this case the energy spectrum of the system is linear. Hence, the spacing between the adjacent levels is fixed to a constant. Interestingly, a non-zero nonlinearity enters in the energy spectrum as we go beyond the quadratic potential, and the exponent takes a value other than $k=2$. Therefore, for the sake of clearity, we define a nonlinearity-measure parameter $\rho$, and redefine the power law exponent, $k$, as $k=2+\rho$. It is obvious that for the quadratic potential, we find $\rho=0$. Hence, the parameter $\rho$ defines the extent of nonlinearity in a system.

From Eqs. (10) and (11) we note that in addition to the power-law exponent $k$, frequency $\omega$ and nonlinearity $\zeta$, have important dependence on mean quantum number $\bar{n}$, and the effective Planck's constant $k$. Hence, the general relations for the frequency, $\omega$, and the nonlinearity, $\zeta$ become

$$
\omega=\frac{1}{k} \frac{2(2+\rho)}{(4+\rho)}\left(\bar{n}+\frac{\gamma}{4}\right)^{-1} E_{\bar{n}}^{(\rho)},
$$

and

$$
\zeta=\frac{1}{2 k^{2}} \frac{\rho(2+\rho)}{(4+\rho)^{2}}\left(\bar{n}+\frac{\gamma}{4}\right)^{-2} E_{\bar{n}}^{(\rho)} .
$$

In terms of the nonlinearity measure $\rho$, the Eqs. (22) and (23) lead us to calculate the recurrence times, $T_{\lambda}^{(c l)}$ and $T_{\lambda}^{(Q)}$, given in Eqs. (18) and (19) in the presence of external modulation. We express these times as

$$
T_{\lambda}^{(c l)}=\left\{1+\frac{1}{2}\left(\frac{\lambda V \rho \Delta^{2}}{2(2+\rho) E_{\bar{n}}^{(\rho)}}\right)^{2} \frac{1}{\left(1-\mu^{2}\right)^{2}}\right\} T_{0}^{(c l)} \Delta,
$$


and

$$
T_{\lambda}^{(Q)}=\left\{1-\frac{1}{2}\left(\frac{\lambda V \rho \Delta^{2}}{2(2+\rho) E_{\bar{n}}^{(\rho)}}\right)^{2} \frac{3+\mu^{2}}{\left(1-\mu^{2}\right)^{3}}\right\} T_{0}^{(Q)} .
$$

Here, we have

$$
T_{0}^{(c l)}=\frac{\pi k}{E_{\bar{n}}^{(\rho)}} \frac{(4+\rho)}{(2+\rho)}\left(\bar{n}+\frac{\gamma}{4}\right)
$$

and

$$
T_{0}^{(Q)}=\frac{2 \pi k}{E_{\bar{n}}^{(\rho)}} \frac{(4+\rho)^{2}}{\rho(2+\rho)}\left(\bar{n}+\frac{\gamma}{4}\right)^{2},
$$

respectively, as the classical period and the quantum revival time in the absence of external modulation. Moreover, the parameter $\mu$ is defined as a function of $\rho$, as

$$
\mu=\frac{N \rho \Delta}{2(4+\rho)\left(\bar{n}+\frac{\gamma}{4}\right)} .
$$

Quadratic Potential: As discussed above, for the quadratic potential we find the nonlinearity-measure parameter $\rho=0$, and threfore the energy spectrum is linear. From Eqs. (24) and (25), we note that in this special case, the time scales in the presence of the external modulation and its absence are related as,

$$
\begin{aligned}
& T_{\lambda}^{(c l)}=T_{0}^{(c l)} \Delta, \\
& T_{\lambda}^{(Q)}=T_{0}^{(Q)} .
\end{aligned}
$$

Interestingly, the Eq. (27) leads us to conclude that in the quadratic potential case, $T_{0}^{(Q)}=\infty$, therefore, quantum revival in the absence and in the presence of the external modulation occurs after an infinite time, whereas the classical period is constant and independent of $\bar{n}$. Hence, the quantum 
evolution of a particle undergoing harmonic motion in the quadratic potential, mimics the classical evolution and shows a recurrence after a classical period only.

The nonlinearity measure $\rho$ may take positive or negative values. In our later discussion, we consider two possible cases on $\rho$ : First, when $\rho$ is positive which corresponds to tightly binding potentials; Second, when $\rho$ is negative which expresses loosely binding potentials. Thus, the quadratic potential provides a boundray between the two kind of potentials.

\section{Tightly Binding Potentials}

In case of tightly binding potentials, the nonlinearity measure $\rho$, is positive. As the value of $\rho$ increases slightly beyond zero, the energy spectrum exhibits a weak nonlinearity, which we may express as $\rho=+\epsilon$. From Eq. (24), we note that for the weak nonlinearity case, the classical period in the presence of the external modulation, $T_{\lambda}^{(c l)}$, becomes larger than its value in the absence of the nonlinearity. However, Eq. (25) explains that the corresponding value of quantum revival time, $T_{\lambda}^{(Q)}$, starts reducing from its value present in the linear case. Hence, from Eqs. (24) and (25) we note that, as the value of

$\rho$ increases, the value of $T_{\lambda}^{(c l)}$ goes on increasing. However, $T_{\lambda}^{(Q)}$ shows a decrease, so far as $\mu^{2}<1$.

For the smaller values of $\rho$, we find that the classical period and the quantum revival time in the presence and in the absence of external driving 
force are related as

$$
\frac{3 T_{\lambda}^{(c l)}}{4 T_{0}^{(c l)}}+\frac{T_{\lambda}^{(Q)}}{4 T_{0}^{(Q)}}=1
$$

This explains that the decrease in quantum revival time is three times more as compared with the increase in the classical period in the presence of external modulation [19].

At asymptotically large values of $\rho$, we note that the recurrence times in the presence and in the absence of the external modulation becomes independent of $\rho$. In the absence of the modulation the classical period $T_{0}^{(c l)}$, as given in (26), is inversely proportional to the mean quantum number $\bar{n}$, such that, $T_{0}^{(c l)} \propto(\bar{n}+\gamma / 4)^{-\frac{\rho}{4+\rho}}$. However, $T_{0}^{(Q)}$, displays a different dependence on the mean quantum number, $\bar{n}$. From Eq. (27), we find that the quantum revival time is directly proportional to $\bar{n}$, such that, $T_{0}^{(Q)} \propto(\bar{n}+\gamma / 4)^{+\frac{4}{4+\rho}}$. As $\rho$ becomes infinite we note that $T_{0}^{(Q)}$ becomes constant for a constant value of $k$ and independent of $\bar{n}$, as we find in one dimensional box [31].

It is interesting to note that, from Eq. (28), the parameter $\mu$ and the mean quantum number $\bar{n}$ are inversely proportional to each other. Hence, in tightly binding potentials for a smaller (larger) value of $\bar{n}$, we find larger (smaller) value for $\mu$. Equation (25), leads to the conclusion that the quantum revival time $T_{\lambda}^{(Q)}$, increases for the smaller $\bar{n}$, and reduces for the larger $\bar{n}$ in the presence of external modulation as compared with the quantum revival time for the time independent system. However, the classical period, $T_{\lambda}^{(c l)}$, demonstrate always an increase with the modulation strength, as we find from Eq. (24). 
For fixed values of $\rho$, and $\bar{n}$, we note that the recurrence times change as a function of effective Planck's constant, $k$. The Eqs. (26) and (27), indicate that the times $T_{0}^{(c l)}$, and $T_{0}^{(Q)}$, are inversely proportional to $k^{-\rho /(4+\rho)}$. Hence, in potentials with very weak non-linearity, we find that the value of the Planck's constant does not contribute appreciably to recurrence times. However, for large value of $\rho$ the recurrence times in the absence of modulation depend inversely on $k$.

\section{Loosely binding potentials}

For loosely binding potentials the nonlinearity-measure parameter, $\rho$, is negative, therefore, we may express the exponent $k$ as $k=2-\rho$. As discussed earlier, in this case the energy spacing between adjacent levels reduces as the principle quantum number $n$ increases. We express a weak nonlinearity in the energy spectrum by assigning a small value to the nonlinearity-measure parameter. The classical period $T_{\lambda}^{(c l)}$, and the quantum revival time $T_{\lambda}^{(Q)}$, show the same behavior as we find in case of tightly binding potentials: The classical period $T_{\lambda}^{(c l)}$ increases whereas, the quantum revival time $T_{\lambda}^{(Q)}$ decreases as the external modulation strength $\lambda$ grows. However, for fixed values of $\bar{n}$ and $k$, the change in recurrence times is larger than what we find in the case of tightly binding potentials. For the smaller values of $\rho$, we find that the classical period and the quantum revival time in the presence and 
in the absence of external driving force are related as

$$
\frac{T_{\lambda}^{(c l)}}{T_{0}^{(c l)}}-\frac{T_{\lambda}^{(Q)}}{4 T_{0}^{(Q)}}=0 .
$$

A special situation occur at $\rho=2$ at which classical period and the quantum revival time in the presence and in the absence of an external modulation become infinite. From Eq. (1) we find that in this case the potential energy is constant and independent of position. This corresponds to the case of a free particle moving with a kinetic energy and a constant potential energy.

In contrast to the tightly binding potentials, weakly binding potentials display a direct proportionality between the mean quantum number $\bar{n}$ and the classical period $T_{0}^{(c l)}$ in the absence of modulation, such that, $T_{0}^{(c l)} \propto$ $(\bar{n}+\gamma / 4)^{\frac{\rho}{4-\rho}}$. For the reason, they display a larger classical period and consequently a smaller frequency as $\bar{n}$ increases. We find this dependence, for example, in the Fermi accelerator [32].

The quantum revival time in the absence of external modulation, $T_{0}^{(Q)}$, is directly proportional to the mean quantum number, $\bar{n}$, in case of loosely binding potentials, such that, $T_{0}^{(Q)} \propto(\bar{n}+\gamma / 4)^{\frac{4}{4-\rho}}$. The proportionality of $T_{0}^{(c l)}$ and $T_{0}^{(Q)}$ on the mean quantum number $\bar{n}$ indicates that the change of $T_{0}^{(Q)}$, is greater as compared with $T_{0}^{(c l)}$, as the value of $\bar{n}$ varies. Interestingly the ratio between $T_{0}^{(Q)}$ and $T_{0}^{(c l)}$ remains the same both in the tightly binding potentials and loosely binding potentials, however, it is not the same for the times in the presence of an external modulation. 
For fixed values of $\rho$, and $\bar{n}$, in contrast to the tightly binding potentials, we note that $T_{0}^{(c l)}$, and $T_{0}^{(Q)}$, are directly proportional to the effective Planck's constant, $k$. Therefore, for a fixed value of $\bar{n}$ and $\rho$ the recurrence times increase with the scaled Planck's constant, such that, $k^{\rho /(4-\rho)}$.

As an example of loosely binding potentials, we study the linear potential i.e. $k=1$. The energy eigen value of the linear gravitational potential truncated at the origon is given as

$$
E_{n}^{(1)}=\left[\left(n+\frac{\gamma}{4}\right) \frac{3 \hbar \pi}{4 \sqrt{2}} V_{0}\right]^{2 / 3} .
$$

The corresponding frequency $\omega$ is given by

$$
\omega=\frac{2}{3 k}\left(\bar{n}+\frac{\gamma}{4}\right)^{-1} E_{\bar{n}}^{(1)}
$$

and nonlinearity $\zeta$ reads as

$$
\zeta=-\frac{2}{9 k^{2}}\left(\bar{n}+\frac{\gamma}{4}\right)^{-2} E_{\bar{n}}^{(1)}
$$

For linear gravitational potential, in the absence of external modulating force, the classical period is given as

$$
T_{0}^{(c l)}=\frac{3 \pi k}{E_{\bar{n}}^{(1)}}\left(\bar{n}+\frac{\gamma}{4}\right)
$$

and quantum recurrence time is given as

$$
T_{0}^{(Q)}=\frac{18 \pi \hbar}{E_{\bar{n}}^{(1)}}\left(\bar{n}+\frac{\gamma}{4}\right)^{2} .
$$


It is obvious from equations (35) and (36) that classical period and quantum recurrence time are directly proportional to the mean quantum number $\bar{n}$ and varies as $\bar{n}^{1 / 3}$ and $\bar{n}^{4 / 3}$, respectively.

In the presence of external modulating force, the classical period $T_{\lambda}^{(c l)}$ is

$$
T_{\lambda}^{(c l)}=\left\{1+\frac{1}{2}\left(\frac{\lambda V \Delta^{2}}{2 E_{\bar{n}}^{(1)}}\right)^{2} \frac{1}{\left(1-\mu^{2}\right)^{2}}\right\} T_{0}^{(c l)} \Delta,
$$

and quantum revival time reads as

$$
T_{\lambda}^{(Q)}=\left\{1-\frac{1}{2}\left(\frac{\lambda V \Delta^{2}}{2 E_{\bar{n}}^{(1)}}\right)^{2} \frac{3+\mu^{2}}{\left(1-\mu^{2}\right)^{3}}\right\} T_{0}^{(Q)},
$$

where, the value of $\mu$ for gravitational potential reduces to $\mu=-N \Delta / 6\left(\bar{n}+\frac{\gamma}{4}\right)$.

In the presence of external modulation, the classical period and the quantum revival time have been studied for linear potential, which constitutes Fermi accelerator, numerically in the reference $[29,30]$. The numerical results obtained in the earlier work show a very good agreement with the analytical results presented in this paper. The analytical results lead to a better understanding of recurrence tracking microscope (RTM) [21] as well, which is to scan surface structures with nano-meter resolution.

\section{Acknowledgement}

SI wants to thank M. Ali, M. Ayub, R. ul Islam, and K. Naseer for many discussions. He also submit his thanks to HEC for funding through grant 041203721E-026. FS thanks G. Alber, and S. Watanabe for many useful discussions. He also thanks Higher Education Commission, Pakistan (research 
grant R\&D/03/143), Quaid-i-Azam University and Fulbright Foundation, USA, for partial financial assistance. FS thanks Prof. Dr. P. Meystre for his hospitality at the department of Physics, University of Arizona where a part of the work was finished.

\section{References}

[1] J. Parker and C. R. Stroud, Phys. Rev. Lett. 56, 716 (1986).

[2] G. Alber and P. Zoller, Phys. Rep. 199, 231 (1990).

[3] M. Nauenberg, J. A. Yeazell, and C. R. Stroud, Sci. Am. 270, 44 (1994).

[4] A. Ten Wolde, L. D. Noordam, A. Lagendijk and H. B. van Linden van den Heuvel, Phys. Rev. Lett. 61, 2099 (1988).

[5] J. A. Yeazell, M. Mallalieu, J. Parker and C. R. Stroud, Phys. Rev. A 40, 5040 (1989).

[6] M. Nauenberg, J. Phys. B 23, L385 (1990).

[7] R. Bluhm and V. A. Kostelecký, Phys. Rev. A 51, 4767 (1995).

[8] I. Sh. Averbukh, and N. F. Perel'man, Phys. Lett. A 139, 449 (1989).

[9] R. W. Robinett, Phys. Rep. 392, 1 (2004).

[10] J. H. Eberly, N. B. Narozhny, and J. J. Sanchez-Mondragon, Phys. Rev. Lett. 44, 1323 (1980). 
[11] I. Sh. Averbukh, Phys. Rev. A 46, R2205 (1992).

[12] G. Rempe, H. Walther, and N. Klein, Phys. Rev. Lett. 58, 353 (1987).

[13] J. A. Yeazell, M. Mallalieu, and C. R. Stroud, Jr., Phys. Rev. Lett. 64, 2007 (1990).

[14] S. Yu. Grebenshchikov, C. Beck, H. Flöthmann, D. H. Mordaunt and R. Schinke, Chem. Phys. Lett. 271, 197 (1997).

[15] T. Hogg, and B. A. Huberman, Phys. Rev. Lett. 48, 711 (1982).

[16] S. Fishman, D.R. Grempel, R. E. Prange, Phys. Rev. Lett 49, 509 (1982)

[17] F. Saif, Phys. Rev. E 62, 6308 (2000).

[18] F. Saif and M. Fortunato, Phys. Rev. A 65, 013401 (2001).

[19] F. Saif, J. Opt B: Quantum Semiclass. Opt. 7, S116 (2005).

[20] F. Saif, accepted for publication in European Physical Journal D.

[21] F. Saif, Phys. Rev. A 73, (2006).

[22] U. P. Sukhatme, Am. J. Phys. 41, 1015 (1973).

[23] M. M. Nieto, L. M. Simmons Jr., Am. J. Phys. 47, 634 (1979).

[24] E. I. Liboff, Int. J. Theor. Phys. 47, 185 (1979).

[25] R. W. Robinett, J. Math. Phys. 41(4), April (2000). 
[26] M. Born, Mechanics of the atom (Ungar, New York, 1960).

[27] G. P. Berman and G. M. Zaslavsky, Phys. Lett. A 61, 295 (1977).

[28] M. E. Flatté and M. Holthaus, Ann. Phys. (N. Y.) 245, 113 (1996).

[29] F. Saif, J. Phys. Soc. Jap. 69(8), 2363L (2000).

[30] F. Saif, G. Alber, V. Savichev, and W. P. Schleich, J. Opt. B: Quantum Semiclassical Opt. 2, 668 (2000).

[31] Marzoli I., F. Saif, I. Bialynicki-Birula, O. M. Friesch, A. E. Kaplan, W. P. Schleich, Acta Physica Slovaca 48323 (1998).

[32] F. Saif, Phys. Rep. 419, 207 (2005); ibid, Phys. Rep. 425, 369 (2006). 